\title{
Blockers of Wnt3a, Wnt10a, or $\beta$-Catenin Prevent Chemotherapy-Induced Neuropathic Pain In Vivo
}

\author{
Hee Kee Kim ${ }^{1}$ (D) Jingi Bae ${ }^{2}$ - Sung Ho Lee ${ }^{3,4,5}$. Seon-Hee Hwang ${ }^{1}$ - Min-Sik Kim ${ }^{6}$ - Moon Jong Kim ${ }^{3,4,5}$. \\ Sohee Jun ${ }^{3,4,5}$. Chris L. Cervantes ${ }^{3,4,5}$ - Youn-Sang Jung ${ }^{3,4,5}$. Seunghoon Back ${ }^{2}$. Hangyeore Lee ${ }^{2}$. Seung-Eun Lee ${ }^{7}$. \\ Patrick M. Dougherty ${ }^{1}$. Sang-Won Lee ${ }^{2}$. Jae-II Park ${ }^{3,4,5}$. Salahadin Abdi ${ }^{1}$
}

Accepted: 19 October 2020 / Published online: 30 October 2020

(C) The American Society for Experimental NeuroTherapeutics, Inc. 2020

\begin{abstract}
Although chemotherapy is a key cancer treatment, many chemotherapeutic drugs produce chronic neuropathic pain, called chemotherapy-induced neuropathic pain (CINP), which is a dose-limiting adverse effect. To date, there is no medicine that prevents CINP in cancer patients and survivors. We determined whether blockers of the canonical Wnt signaling pathway prevent CINP. Neuropathic pain was induced by intraperitoneal injection of paclitaxel (PAC) on four alternate days in male Sprague-Dawley rats or male Axin2-LacZ knock-in mice. XAV-939, LGK-974, and iCRT14, Wnt/ $\beta$-catenin blockers, were administered intraperitoneally as a single or multiple doses before or after injury. Mechanical allodynia, phosphoproteome profiling, Wnt ligands, and inflammatory mediators were measured by von Frey filament, phosphoproteomics, reverse transcription-polymerase chain reaction, and Western blot analysis. Localization of $\beta$-catenin was determined by immunohistochemical analysis in the dorsal root ganglia (DRGs) in rats and human. Our phosphoproteome profiling of CINP rats revealed significant phosphorylation changes in Wnt signaling components. Importantly, repeated systemic injections of XAV-939 or LGK-974 prevented the development of CINP in rats. In addition, XAV-939, LGK-974, and iCRT14 ameliorated CINP. PAC increased Wnt3a and Wnt10a, activated $\beta$-catenin in DRG, and increased monocyte chemoattractant protein- 1 and interleukin$1 \beta$ in DRG. PAC also upregulated rAxin 2 in mice. Furthermore, $\beta$-catenin was expressed in neurons, including calcitonin generelated protein-expressing neurons and satellite cells in rat and human DRG. In conclusion, chemotherapy increases Wnt3a, Wnt10a, and $\beta$-catenin in DRG and their pharmacological blockers prevent and ameliorate CINP, suggesting a target for the prevention and treatment of CINP.
\end{abstract}

Key Words Chemotherapy-induced neuropathic pain, Wnt ligand, Wnt3a, Wnt10a, $\beta$-catenin, prevention

Hee Kee Kim and Jingi Bae contributed equally to this work.

Supplementary Information The online version contains supplementary material available at https://doi.org/10.1007/s13311-02000956-w.

Hee Kee Kim

hkim9@mdanderson.org

1 Department of Pain Medicine, Division of Anesthesiology, Critical Care \& Pain Medicine, The University of Texas MD Anderson Cancer Center, Houston, TX 77030, USA

2 Department of Chemistry, Center for Proteogenome Research, Korea University, Seoul 02841, Republic of Korea

3 Department of Experimental Radiation Oncology, Division of Radiation Oncology, The University of Texas MD Anderson Cancer Center, Houston, TX 77030, USA
4 Graduate School of Biomedical Sciences, The University of Texas MD Anderson Cancer Center, Houston, TX 77030, USA

5 Program in Genetics and Epigenetics, The University of Texas MD Anderson Cancer Center, Houston, TX 77030, USA

6 Department of New Biology, Daegu Gyeongbuk Institute of Science and Technology (DGIST), Daegu 42988, Republic of Korea

7 Department of Biomedical Science and Technology, Kyung Hee Medical Science Research Institute, Kyung Hee University, Seoul 02447, Republic of Korea 


\section{Introduction}

Chemotherapy-induced chronic neuropathic pain (CINP), a dose-limiting adverse effect of chemotherapy, is produced by various chemotherapeutic drugs, including taxanes (paclitaxel $[\mathrm{PAC}]$ and docetaxel), vinca alkaloids (vincristine and vinblastine), and platinum agents (cisplatin, carboplatin, and oxaliplatin) [1]. CINP is characterized by spontaneous pain conditions and evoked pain conditions, including allodynia [1]. It affects patients at any time during or after treatment and decreases quality of life [1]. Currently, commonly used pain medicines, including opioids, non-steroidal anti-inflammatory drugs, anticonvulsants, and antidepressants, have limited analgesic effects on CINP in patients and animals [1]. In particular, no medicine is effective in preventing CINP [1].

The Wnt pathway plays crucial roles in controlling various cellular processes, including cell proliferation, and human diseases, including cancer $[2,3]$. Wnt signaling also governs the development of nervous tissues, including synapse maturation and plasticity $[4,5]$, and is involved in the regulation of N-methyl-D-aspartate receptors and synaptic plasticity [6]. In the absence of Wnt ligands, $\beta$-catenin, a key mediator of Wnt signaling, is constitutively degraded by the protein destruction complex, which is composed of adenomatous polyposis coli, glycogen synthase kinase 3, and Axin proteins. However, in the presence of Wnt ligands, the binding of Wnt ligands to the Frizzled receptor and LRP5/6 co-receptor activates Dishevelled protein, which inhibits the protein destruction complex. Subsequently, the stabilized $\beta$-catenin protein leads to its nuclear translocation and transactivates Wnt target genes in association with $\mathrm{T}$ cell factors/lymphoid enhancing factor [7]. Among 19 Wnt ligands, Wnt1, Wnt2, Wnt2b, Wnt3a, Wnt8, and Wnt10 induce the stabilization of $\beta$-catenin (canonical Wnt pathway) and transcriptionally upregulate their target genes [8]. The deregulation of $\mathrm{Wnt} / \beta$-catenin signaling is involved in various pathologic conditions, including cancer. Thus, pharmacologic blockers, including XAV939 (inhibitor of $\beta$-catenin stabilization), LGK-974 (inhibitor of Wnt ligand secretion), and iCRT14 (inhibitor of $\beta$-catenin-mediated transactivation), have been tested in preclinical and clinical settings as a therapeutic option for cancer treatment $[2,9]$. In previous studies, several Wnt blockers showed analgesic effects on various neuropathic pain models, including spinal nerve ligation, chronic constriction injury, and CINP models [10-12]. However, the preventive effect of Wnt blockers and the role of Wnt ligands have not been determined. Thus, the aims of this study were to (1) assess the preventive effects of canonical $\mathrm{Wnt} / \beta$-catenin pathway blockers on CINP in rats, (2) identify the role of Wnt ligands of canonical Wnt/ $\beta$-catenin pathway in the dorsal root ganglia (DRGs), and (3) find the location of $\beta$-catenin in DRG in rats and human.

\section{Materials and Methods}

\section{Animals}

We used adult male Sprague-Dawley rats (200-350 g; Harlan Sprague Dawley Company, Houston, TX, USA) and male Axin2-LacZ knock-in mice (20-30 g; Jackson Laboratory, USA). The experimental protocol was approved by the institutional animal care and use committees of The University of Texas MD Anderson Cancer Center (Houston, TX, USA).

\section{Paclitaxel-Induced Neuropathic Pain Model in Rats or Mice}

PAC (GenDepot, Katy, TX, USA) was dissolved in dimethyl sulfoxide (DMSO) at a concentration of $50 \mathrm{mg} / \mathrm{ml}$ and stored in a freezer $\left(-80^{\circ} \mathrm{C}\right)[13-15]$. Before injection, the stock solution was mixed with an equal volume of Tween 80 and then diluted with sterile saline to a concentration of $2 \mathrm{mg} / \mathrm{ml}$ for rats or $0.4 \mathrm{mg} / \mathrm{ml}$ for mice.

For the paclitaxel-induced neuropathic pain (PINP) rats, PAC $(2 \mathrm{mg} / \mathrm{ml})$ was injected intraperitoneally at a dose of $2 \mathrm{mg} / \mathrm{kg} / \mathrm{ml}$ on days $0,2,4$, and 6 [13-15]. Vehicle (VEH) rats received $4 \%$ DMSO and $4 \%$ Tween 80 in sterile saline $(1 \mathrm{ml} / \mathrm{kg}$ ) without PAC.

For the PINP mice, PAC $(0.4 \mathrm{mg} / \mathrm{ml})$ was injected intraperitoneally at a dose of $4 \mathrm{mg} / \mathrm{kg} / 10 \mathrm{ml}$ on days $0,2,4$, and 6 [16]. VEH mice received $0.8 \%$ DMSO and $0.8 \%$ Tween 80 in sterile saline $(10 \mathrm{ml} / \mathrm{kg})$ without PAC.

\section{Measurement of Mechanical Allodynia}

In rats, the mechanical threshold of the left hind paw was determined using the up-down method with a set of von Frey monofilaments and then calculated a $50 \%$ mechanical threshold [13-15, 17]. In mice, the mechanical hypersensitivity of the left hind paw was determined by measuring the foot withdrawal response to $0.1 \mathrm{~g}$ filament and then calculated as a percentage of positive response [18].

\section{Phosphoproteome Profiling of CINP Rats}

The L1-6 DRGs were collected from VEH-injected rats and PAC-injected rats on day 16 or 17 after the first PAC or vehicle injection. In brief, DRG tissue (about $15 \mathrm{mg}$ ) was pulverized, sonicated, and centrifuged, and then the supernatants were collected $[19,20]$. The protein was digested by filteraided sample preparation with trypsin. For tandem mass tag (TMT) labeling, the digested peptide was labeled using 10plex TMT reagents (90111, Thermo Scientific, Rockford, IL). The labels for the samples were three vehicle peptides (126, $128 \mathrm{~N}$, and $129 \mathrm{C}$ tags) and three PAC peptides $(127 \mathrm{~N}, 128 \mathrm{C}$, and $130 \mathrm{~N}$ tags). The TMT-labeled peptides were used for 
phosphopeptide enrichment. The labeled peptides were divided into the $\mathrm{Fe}^{3+}$-NTA beads, where the phosphopeptides were enriched. The phosphopeptides were analyzed through an online, two-dimensional, non-contiguous concatenating, and fractionating reverse-phase/reverse-phase liquid chromatography system [21]. The eluted phosphopeptides were ionized using an in-house nano-electrospray source. The mass spectrometer was set to analyze peptide ions in data-dependent acquisition mode. The MS/MS scans were acquired at a resolution of 35,000 . The automated gain control target value and the maximum ion injection time were set to $2.0 \times 10^{5}$ and $120 \mathrm{~ms}$, respectively.

\section{Western Blot Analysis}

The L1-6 DRGs were collected from VEH-injected rats, PACinjected rats, and XAV-treated PAC-injected rats on day 16 or 17 after the first PAC or vehicle injection. The DRGs were homogenized in RIPA cell lysis buffer and centrifuged. The supernatant was loaded and transferred to membranes [15]. The blots were incubated with primary antibody against phosphorylated nuclear factor- $\mathrm{KB}$ (phospho-NF- $\mathrm{kB}$, rabbit, 1:1000, $65 \mathrm{kDa}$, Cell Signaling Technology), monocyte chemoattractant protein-1 (MCP-1, rabbit, 1:500, $12 \mathrm{kDa}$, Santa Cruz Biotechnology), interleukin-1 $\beta$ (IL-1 $\beta$, rabbit, 1:1000, 17/31 kDa, Santa Cruz Biotechnology), and GAPDH (rabbit, 1:5000, $37 \mathrm{kDa}$, Santa Cruz Biotechnology) overnight at $4{ }^{\circ} \mathrm{C}$. The blots were then incubated with the anti-rabbit horseradish peroxidase-conjugated secondary antibody (1:5000, GenDepot). The immunoblots were analyzed with a chemiluminescence detection system and normalized to GAPDH.

\section{Reverse Transcription-Polymerase Chain Reaction (RT-PCR)}

The L1-6 DRGs were collected from VEH-injected rats, PACinjected rats, or XAV-treated PAC-injected rats on day 16 or 17 after the first PAC or vehicle injection. The L1-6 DRGs were extracted with TRIzol for RNA extraction. RNA $(1 \mu \mathrm{g})$ was used for cDNA synthesis using the iScript cDNA synthesis kit (Biorad). Semi- and quantitative real-time PCR (qRTPCR) were performed using a PCR machine (Applied Biosystems) with the specific primers listed in Table 1. Target gene expression was normalized using two endogenous control genes (Actb and Hprt1). Comparative $2^{-\Delta \Delta \mathrm{Ct}}$ methods were used to quantify the qRT-PCR results.

\section{X-Gal (5-Bromo-4-Chloro-3-Indolyl- $\beta$-D-Galacto- Pyranoside) Staining}

DRG samples from Axin2-LacZ knock-in mice, a Wnt signaling reporter strain, were fixed with $2 \%$ formaldehyde containing $0.2 \%$ glutaraldehyde for $10 \mathrm{~min}$, rinsed with phosphate-buffered saline (PBS), and incubated with staining solution $\left(2 \mathrm{mM} \mathrm{MgCl}_{2}, 4 \mathrm{mM} \mathrm{K}_{3} \mathrm{Fe}(\mathrm{CN})_{6}, 4 \mathrm{mM} \mathrm{K}_{4} \mathrm{Fe}(\mathrm{CN})_{6}\right.$, and $1 \mathrm{mg} / \mathrm{ml} \mathrm{X}$-gal) for $2 \mathrm{~h}$ at $37^{\circ} \mathrm{C}$. The stained samples were post-fixed and analyzed.

\section{Hematoxylin-and-Eosin Staining}

Tissues were collected from rats and then treated with formalin and paraffin; $0.5-\mu \mathrm{m}$ slices were stained with hematoxylin and eosin. Images were obtained using Axio Observer and AxioVision microscopes (Zeiss).

\section{Immunohistochemical Analyses of Rat DRG}

The L5 DRGs were collected from vehicle rats $(N=3)$ and PAC rats $(N=3)$ on day 16 after the first PAC or vehicle injection [22]. The L5 DRGs were fixed, cryosectioned, and mounted on microscope slides. The sections were incubated with combinations of the primary antibodies to non-phospho active $\beta$-catenin (marker for canonical Wnt signaling pathway, rabbit, 1:100, Cell Signaling Technology), NeuN (neuron marker, mouse, 1:50, Abcam Cambridge), calcitonin gene-related peptide (CGRP; peptidergic somatosensory neuronal marker, mouse, 1:50, Santa Cruz Biotechnology), or glial fibrillary acidic protein (GFAP; satellite cell marker, mouse, 1:100, Santa Cruz Biotechnology) overnight at $4{ }^{\circ} \mathrm{C}$ and then incubated with secondary antibodies with Alexa Fluor 568 or Alexa Fluor 488 (goat anti-rabbit with Alexa Fluor 488, 1:100, GenDepot or goat anti-mouse with Alexa Fluor 594, 1:100, GenDepot) [22]. In addition, ProLong Diamond Antifade Mountant was applied to the sections, with 4',6diamidino-2-phenylindole (DAPI; nuclear and chromosome marker). The stained tissue sections were viewed under a Vectra 2 microscope from PerkinElmer.

\section{Immunohistochemical Analyses of Human DRG}

We collected DRGs from patient donor at MD Anderson Cancer Center who provided legal written consent. This protocol was approved by MD Anderson Cancer Center and the patient. One patient had a history of cancerrelated neuropathic pain that appeared to be multifactorial in etiology, including direct neural compression and the physiological effects of the peritumoral environment, which were due to radiation therapy involving the spine. During standard-of-care surgical resection of metastatic oncological disease, which involved ligation of spinal nerve roots, this patient donated two DRGs: one from the pain area and the other from a non-pain area [23]. Immediately after each ganglion was excised in the operating room, it was cut with a sterile scalpel, fixed, 
Table 1 Primer information for semi-quantitative RT-PCR

\begin{tabular}{|c|c|c|}
\hline Rat gene & Forward ( $5^{\prime}$ to $\left.3^{\prime}\right)$ & Reverse (5' to $\left.3^{\prime}\right)$ \\
\hline Wnt1 & ATAGCCTCCTCCACGAACCT & GGAATTGCCACTTGCACTCT \\
\hline Wnt2 & GAAGAAAGGGAGTGGCAAGGAC & GCATCTACAAATGCACGGGCGAA \\
\hline$W n t 2 b$ & ACACCAGTTCCGTCATCACC & CCCGAGTGATAGCATGGACC \\
\hline Wnt3 & ACCTGGAGAAGGCTGGAAAT & ATGTGATCCAGGATGGTCGT \\
\hline Wnt $3 a$ & TGCAAATGCCACGGACTATC & AGACTCTCGGTGTTTCTCTACC \\
\hline Wnt4 & CTGGAGAAGTGTGGCTGTGA & GGACTGTGAGAAGGCTACGC \\
\hline Wnt5a & TGGCCACATTTTTCTCCTTC & GCAGAGAGGCTGTGCTCCTA \\
\hline$W n t 5 b$ & GCAGGGTCATGCAGATAGG & CGGTAGCCATACTCCACGT \\
\hline Wnt6 & GCGGTCACTCAGGCCTGTT & GGGTGCCTGACAACCACACT \\
\hline Wnt7a & CCCGAACCCTCATGAACTTA & TGTGGTCCAGCACGTCTTAG \\
\hline$W n t 7 b$ & AGCCAACATCATCTGCAACA & GGCATTCATCGATACCCATC \\
\hline Wnt $8 a$ & CCTGGGAGCGGTGGAACT & CCTGGTGTGGGTTGAAAACTG \\
\hline Wnt $8 b$ & GTGGCTGTGATGACTCCCGA & AACTGCTTGGAAATTGCCTCTC \\
\hline Wnt9a & TCTAACCCTGGAGACCGAGG & GGAACTGGTACTGGCACTCC \\
\hline Wnt $9 b$ & CACCCATGTGGGCATCAA & CCATGACACTTGCAGGTTGTTC \\
\hline Wnt10a & TCACTCCGACCTGGTCTACTT & CTCAGTGATGCGGCATTCTTC \\
\hline Wnt10b & CCTCAAGCGCGGTTTCC & CAGCAGCCAGCATGGAGAA \\
\hline Wnt11 & GGGAGTCAGCCTTCGTGT & GGTTGTCCGCACATCCTC \\
\hline Wnt16 & TGGCTGTAACCTCCTCTGCT & GAGGCAATCTCATGCTAGGC \\
\hline Hprt1 & TCTTTGCTGACCTGCTGGAT & CCCCCGTTGACTGGTCATTA \\
\hline
\end{tabular}

cryoprotected, and mounted. The sections were stained with the primary and secondary antibodies as described above in the "Immunohistochemical Analyses of Rat DRG" section.

\section{Sedation}

Sedation testing was based on 5-point scales of posture $(0=$ normal, 4 = flaccid atonia) and righting reflexes ( $0=$ struggles, $4=$ no movement) [15]. Sedation was assessed immediately after the pain test.

\section{Release of Wnt Ligands from the Primary DRG Cell Culture}

The lumbar DRGs were collected from rats and cultured in a $\mathrm{CO}_{2}$ incubator [15]. To measure the release of Wnt ligands, we cultured DRG cells in a chambered coverglass in DMEM with 5\% FBS. After a 24-h incubation, cells were treated with vehicle or PAC for $24 \mathrm{~h}$. The $r W n t 3 a$ and $r W n t 10 a$ levels in treated DRG cells were measured by qRT-PCR.

\section{Administration of XAV-939, LGK-974, and iCRT14}

XAV-939, LGK-974, and iCRT14 (MedChemExpress, Monmouth Junction, NJ, USA) were dissolved in DMSO and suspended in $1 \%$ sodium CMC in PBS without calcium and magnesium. XAV-939, LGK-974, and iCRT14 were administered via intraperitoneal injection.

\section{Administration of XAV-939}

To determine the single and extended analgesic effects of XAV-939, we administered a single intraperitoneal injection $(0.3,1$, or $3 \mathrm{mg} / \mathrm{kg})$ on day 16 and multiple intraperitoneal injections ( $3 \mathrm{mg} / \mathrm{kg}$ ) twice daily for 4 days (Table 2). To determine the preventive effects, we administered multiple intraperitoneal injections of XAV-939 (3 mg/kg twice daily during days 6-14) or a vehicle group.

\section{Administration of LGK-974}

To determine the single and extended analgesic effects of LGK-974, we administered a single intraperitoneal injection $(0.5$ or $2 \mathrm{mg} / \mathrm{kg})$ on day 16 and multiple intraperitoneal injections of $2 \mathrm{mg} / \mathrm{kg}$ twice daily for 4 days (Table 2 ). To determine the preventive effects, the LGK-974 group (2 mg/kg twice daily) was intraperitoneally injected during days 6-14.

\section{Administration of iCRT14}

To determine the single analgesic effects of iCRT14, a single intraperitoneal injection $(10,30 \mathrm{mg} / \mathrm{kg})$ of iCRT14 was intraperitoneally injected on day 16 (Table 2). 
Table 2 Intraperitoneal injections of XAV-939, LGK-974, and iCRT14 in rats

\begin{tabular}{llll}
\hline Name & Vehicle & Purpose & Dose and schedule \\
\hline XAV-939 & 3\% DMSO in 1\% sodium carboxymethyl-cellulose (CMC) in PBS & Single analgesic effects & $0.3,1$, and 3 mg/kg on day 16 \\
& & Extended analgesic effects & $3 \mathrm{mg} / \mathrm{kg}$ twice daily on days 16-19 \\
& & Preventive effects & $3 \mathrm{mg} / \mathrm{kg} \mathrm{twice} \mathrm{daily} \mathrm{on} \mathrm{days} \mathrm{6-14}$ \\
LGK-974 & $1 \%$ DMSO in 1\% CMC in PBS & Single analgesic effects & $0.5 \mathrm{and} 2 \mathrm{mg} / \mathrm{kg}$ on day 16 \\
& & Extended analgesic effects & $2 \mathrm{mg} / \mathrm{kg}$ twice daily on days 16-19 \\
& & Preventive effects & $2 \mathrm{mg} / \mathrm{kg}$ twice daily on days 6-14 \\
iCRT14 & $10 \%$ DMSO in 1\% CMC in PBS & Single analgesic effects & 10 and 30 mg/kg on day 16 \\
\hline
\end{tabular}

\section{Statistical Analyses}

Data were summarized as means with standard errors or standard deviation for behavioral testing, Western blot analysis, and qRT-PCR. The data were analyzed using GraphPad Prism 6 software and two-way repeated measures analyses of variance with one repeated factor (time), followed by the Tukey post hoc test for behavioral testing or the Mann-Whitney $U$ test for Western blot analysis and qRT-PCR. In all cases, $P<0.05$ was considered statistically significant.

\section{Results}

\section{Phosphoproteomic Analysis of PAC-Treated Rat DRG}

In the phosphoproteomic experiments from rat DRG (Fig. 1(a)), the data included 18,197 distinct phosphopeptides (22,701 phosphosites) that were mapped on 5878 phosphoproteins. Phosphorylation (STY) sites of DRG are shown in supplemental Table S1. PAC upregulated 1410 phosphorylations and downregulated 1006 phosphorylations $(n=3$ for the PAC and vehicle groups, $p<0.1$ ) (Fig. 1(b, c)). A gene set enrichment analysis (GSEA) using phosphorylations $(p \leq 0.1)$ showed marked enrichment in Wnt signaling pathway $(\underline{n}=3$ for the PAC and vehicle groups, $p \leq 0.05$ ) (Fig. 1(d)). Intriguingly, PAC upregulated the phosphorylation of many canonical Wnt signaling components, including Camk2D, Apc, Csnk1d, Csnk1e, Csnk1g2, GSK3 $\beta$, and Axin1, and downregulated the phosphorylation of Apc (S2609) (Fig. 1(e)). Of note, S9 and Y 216 of GSK3 $\beta$ were hyperphosphorylated in PAC-treated DRG (Fig. 1(f)). The data suggest that PAC upregulated the phosphorylation of the canonical Wnt signaling pathway.

\section{Analgesic Effects of Pharmacological Blockers}

In pain behavioral testing, sedation produced false analgesic effects. All rats treated with XAV939, LGK-974, and iCRT14 or vehicle had a score of 0 on the basis of both the posture and righting reflex scales, indicating that they did not produce sedation. In addition, XAV-939 did not affect the overall integrity of the small intestine, crypts, liver, and skin (Fig. S1).

A single injection of XAV-939 significantly increased the mechanical threshold in rats at doses of $0.3,1$, and $3 \mathrm{mg} / \mathrm{kg}$ $(n=6, p<0.05$, Fig. 2(a)). The $3 \mathrm{mg} / \mathrm{kg}$ significantly increased the mechanical threshold $(6.8 \mathrm{~g}$ at $1.5 \mathrm{~h}, p<0.05)$ compared with VEH, but the threshold had returned to the baseline at $4 \mathrm{~h}(n=6)$. The repeated injections of XAV-939 ( $3 \mathrm{mg} / \mathrm{kg}$ twice daily on days 16-19) significantly increased the mechanical threshold on days 17-20 $(p<0.05)$, and the threshold returned to the baseline on day $21(n=6$, Fig. 2(b)). These results indicate that the inhibitor of $\beta$-catenin stabilization produces analgesic effects.

A single injection of LGK-974 significantly increased the mechanical threshold in rats at doses of 0.5 and $2 \mathrm{mg} / \mathrm{kg}(n=$ $6, p<0.05$, Fig. 2(c)). Two milligrams/kilogram of LGK-974 significantly increased the threshold $(7.6 \mathrm{~g}$ at $1.5 \mathrm{~h}, p<0.05)$ compared with the VEH group, but the threshold had returned to the baseline at $4 \mathrm{~h}(n=6)$. The repeated injections of LGK$974(2 \mathrm{mg} / \mathrm{kg}$ twice daily on days 16-19) significantly increased the threshold on days 17-20 $(p<0.05)$, and the threshold returned to the baseline on day $21(n=6$, Fig. 2(d)). These results indicate that the inhibitor of Wnt ligand secretion produces analgesic effects.

A single injection of iCRT14 significantly increased the threshold in rats at doses of 10 and $30 \mathrm{mg} / \mathrm{kg}(n=6$, Fig. 2(e)). Thirty milligrams/kilogram of iCRT14 significantly increased the threshold $(5.0 \mathrm{~g}$ at $1.5 \mathrm{~h}, p<0.05)$ compared with the vehicle group $(n=6)$. These data indicate that the inhibitor of $\beta$-catenin-mediated transactivation produces analgesic effects.

\section{Preventative Effects of Pharmacological Blockers}

The VEH decreased the mechanical threshold from normal on day 6 ; it reached the lowest mechanical threshold $(\sim 1 \mathrm{~g})$ on day 14 (Fig. 2(f)). However, XAV-939 (3 mg/kg twice daily from analgesic effects, on days 6-14) did not change the mechanical threshold (more than $10 \mathrm{~g})$ by day $40(n=6, p<0.05$, Fig. 2(f)). In addition, LGK-974 (2 mg/kg twice daily on days 6-14) did not change the mechanical threshold by day $40(n=$ 


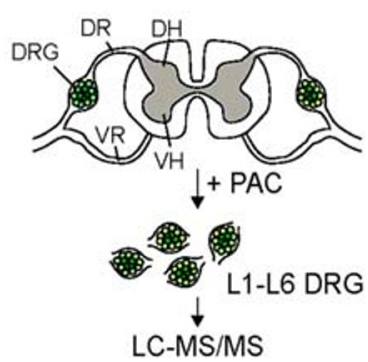

d Wnt signaling pathway

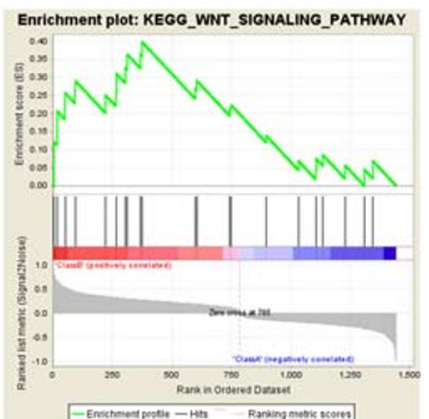

Fig. 1 Phosphoproteomics profiling of DRG. Paclitaxel (PAC, $2 \mathrm{mg} / \mathrm{kg}$, $n=3$ ) or vehicle (4\% dimethyl sulfoxide and $4 \%$ Tween 80 in saline, $1 \mathrm{ml} / \mathrm{kg}, n=3$ ) was injected intraperitoneally on days $0,2,4$, and 6 in the PAC and vehicle groups. (a) Overall scheme describing sample preparation, phosphor-proteome profiling, and data analysis. On day 16 after the first PAC or vehicle injection, the L1-6 DRG were obtained, homogenized, lysed, digested with trypsin, and labeled with TMT. TMT-labeled peptides were fractioned, immobilized, enriched with metal-affinity

$6, p<0.05$, Fig. $2(\mathrm{~g}))$. These results strongly suggest that the inhibitor of $\beta$-catenin stabilization and inhibitor of Wnt ligand secretion prevent PINP.

\section{PAC-Induced Activation of Wnt/ $\beta$-Catenin Signaling in the DRG}

This injection of PAC ( $2 \mathrm{mg} / \mathrm{kg}$ on days $0,2,4$, and 6$)$ in rats did not significantly affect microtubule assembly in the DRG (Fig. 3 (a, b)). However, DRG neuronal cells from PAC rats exhibited active $\beta$-catenin, as represented by the overall increased level and nuclear localization of active $\beta$-catenin $(n=$ $3, p<0.05$, Fig. 3 (c, d)). XAV-939 decreased PAC-increased active $\beta$-catenin level $(n=3$, Fig. 3 (c, d)).

PAC injection increased Axin2-LacZ expression in Axin2$L a c z$ mice, as visualized by $\beta$-catenin in vivo by $\mathrm{X}$-gal staining $(n=3$, Fig. $3(\mathrm{e}, \mathrm{f}))$ [24]. Consistently, PAC administration upregulated rAxin2, as determined by qRT-PCR, which was restored by the intraperitoneal injection of XAV939 $(n=3$, Fig. $3(\mathrm{~g}))$. PAC induced neuropathic pain behavior in Axin2-LacZ mice ( $n=3$ for PAC and $n=2$ for vehicle, Fig. $3(j))$ in a similar manner to that in rat PINP models (Fig. 2).

Furthermore, the expression of canonical Wnt ligands, including rWnt3, rWnt3a, and rWnt10a, was higher in PAC- chromatography phosphopeptide, and analyzed with LC-MS/MS systems. DR, dorsal root; VR, ventral root; $\mathrm{DH}$, dorsal horn; and VH, ventral horn. (b) Of the 22,701 phosphopeptides identified, 2416 were differentially expressed, with statistical significance $(n=3, p \leq 0.1)$. (c) Volcano plot for all phosphorylated proteins $(n=3)$. (d) Enrichment plot of the Wnt signaling pathway $(n=3)$. (e) Phosphorylated sites of canonical Wnt signaling-related proteins $(n=3)$. (f) The graph shows the position of the phosphorylated GSK3 $\beta$

treated DRG than in VEH samples $(n=3$, Fig. $3(\mathrm{~h}))$. Similarly, PAC-treated primary cultured DRG neuronal cells significantly increased the level of $\mathrm{rWnt} 3 \mathrm{a}(N=3, p=0.0008)$ and rWnt10a $(n=3, p=0.0105$, Fig. 3(i)). These results indicate that $\mathrm{PAC}$ activates $\mathrm{Wnt} / \beta$-catenin signaling, including Wnt3a, Wnt10a, and $\beta$-catenin in the DRG.

\section{PAC-Induced Inflammatory Cytokines in the DRG}

The levels of phosphorylated nuclear factor- $\mathrm{kB}$ (phosphoNF- $\mathrm{kB}$ ), monocyte chemoattractant protein-1 (MCP-1), and interleukin-1 $\beta$ (IL-1 $\beta$ ) in the PAC group were $2.5,1.9,1.5$, and 1.5 times higher, respectively, than were those in the VEH group, as determined by a Western blot analysis $(n=3$, $p<0.05$, Fig. $3(\mathrm{k}-\mathrm{m})$ ). The subsequent administration of XAV-939 decreased the protein levels of phospho-NF-KB, MCP-1, and IL- $1 \beta$ in the PAC group $(n=3$, Fig. $3(\mathrm{k}-\mathrm{m}))$. These data indicate that the protein levels of inflammatory cytokines in the DRG were increased by PAC and decreased by XAV-939.

\section{Co-localization of $\beta$-Catenin in Rat DRGs}

Active $\beta$-catenin was expressed in the L5 DRGs of both vehicle- and PAC-treated rats (VEH for Fig. 4(a-d), PAC 


\section{[1] Analgesic effects of pharmacological Wnt blockers}
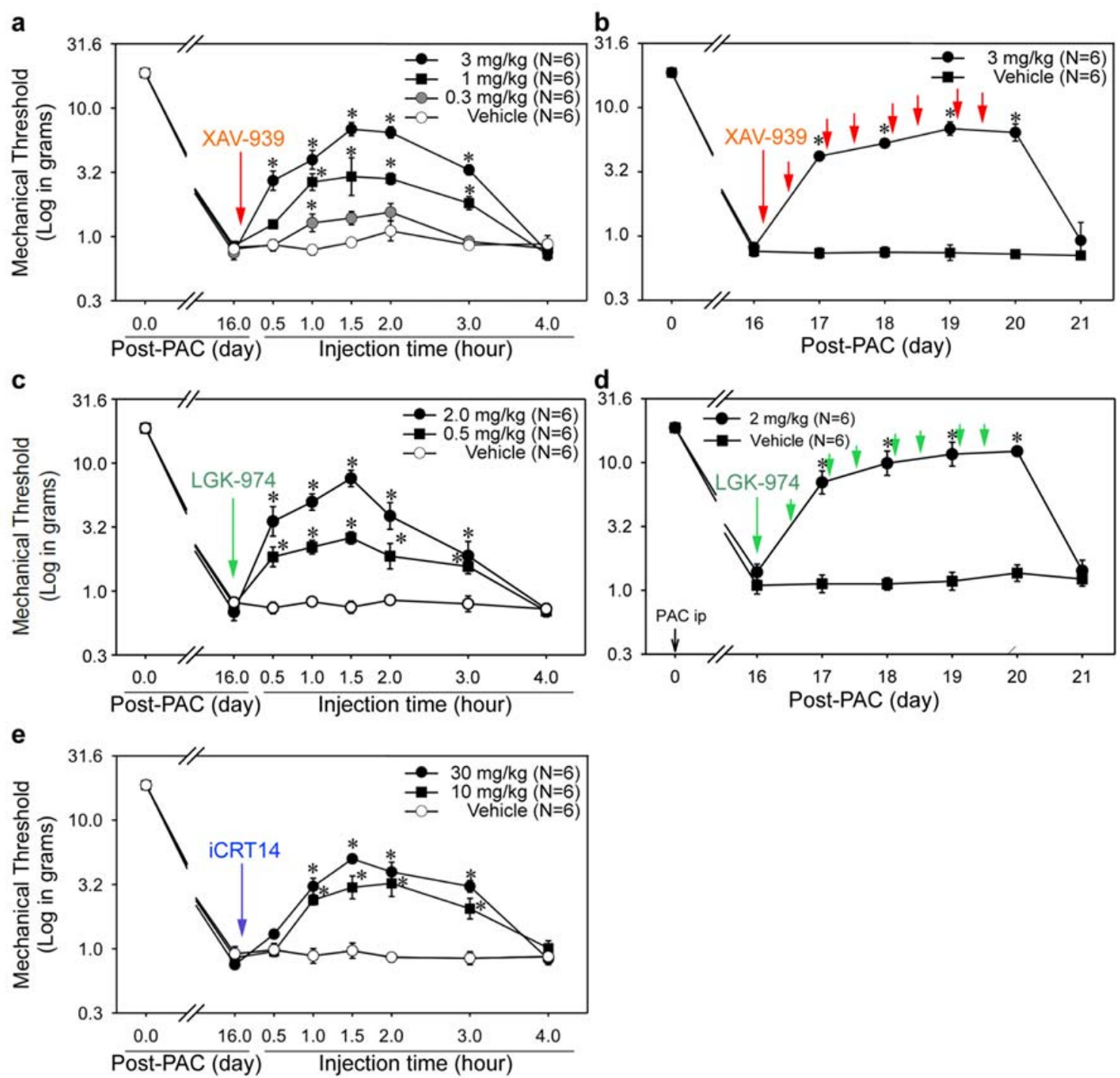

\section{[2] Preventive effects of pharmacological Wnt blockers}

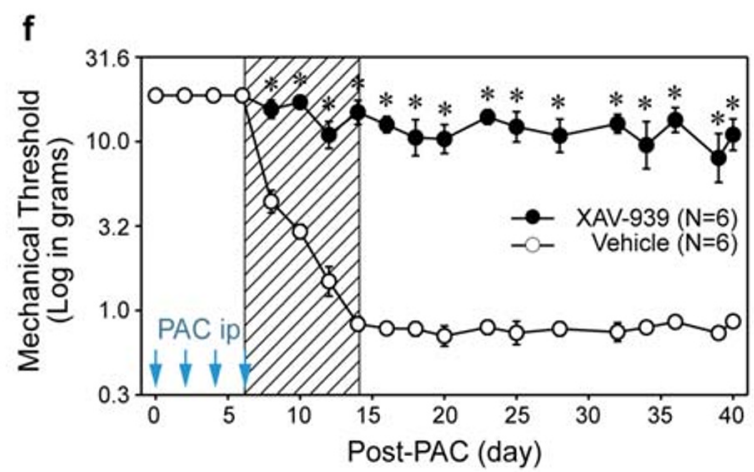

for Fig. 4(e-p)). The intensity of $\beta$-catenin in PAC was higher than that in VEH $(n=3, p<0.05$, Fig. 4(a, e, q)). The expression of $\beta$-catenin in PAC was localized with the neuron (Fig. 4(h)), including the CGRP-expressing neuron

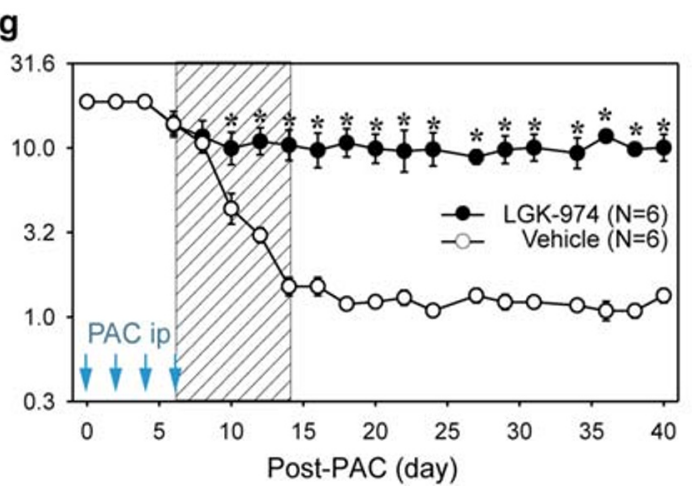

(Fig. 4(1)) and GFAP-expressing satellite cells (Fig. 4(p)). These data indicate that $\beta$-catenin was co-localized in rat DRG neurons, including CGRP-expressing cells and satellite cells. 
Fig. 2 Analgesic and preventive effects of $\mathrm{Wnt} / \beta$-catenin blockers on PINP in rats. (1) Analgesic effects of pharmacological Wnt blockers. (a) PAC was injected intraperitoneally on days $0,2,4$, and 6 , and the mechanical threshold was measured. Each blocker was dissolved in DMSO and then suspended in 1\% CMC in PBS (vehicle). On day 16, 24 rats were divided into four groups that received an injection of vehicle, $0.3,1$, or $3 \mathrm{mg} / \mathrm{kg}$ of XAV-939 ( $n=6$, arrow). Note that the $3 \mathrm{mg} / \mathrm{kg}$ of XAV939 significantly increased the mechanical threshold for $3 \mathrm{~h}(n=6$, $p<0.05$ ). (b) On day 16, 12 rats were divided into two groups that received an injection of vehicle or $3 \mathrm{mg} / \mathrm{kg}$ of XAV-939 twice daily for 4 days during days $16-19(n=6)$. The multiple injections of XAV-939 significantly increased the mechanical threshold on day 17 , and the threshold remained considerably higher than that in the vehicle group for 4 days $(n=6, p<0.05)$. (c) On day 16,18 rats were divided into three groups that received an injection of vehicle, 0.5 , or $2 \mathrm{mg} / \mathrm{kg}$ of LGK-974 ( $n=6$, arrow). The $2 \mathrm{mg} / \mathrm{kg}$ of LGK-974 significantly increased the mechanical threshold for $3 \mathrm{~h}$ compared with that in the vehicle group $(n=6$, $p<0.05$ ). (d) On day 16, 12 rats were divided into two groups that received an injection of vehicle or $2 \mathrm{mg} / \mathrm{kg}$ of LGK-974 twice daily for 4 days during days 16-19 $(n=6)$. The multiple injections of LGK-974 significantly increased the mechanical threshold on day 17 , and the threshold remained considerably higher than that in the vehicle group for 4 days $(n=6, p<0.05)$. (e) On day 16,18 rats were divided into three groups that received an injection of vehicle, 10 , or $30 \mathrm{mg} / \mathrm{kg}$ of iCRT14 $(n=6$, arrow). The $30 \mathrm{mg} / \mathrm{kg}$ of iCRT14 significantly increased the mechanical threshold for $3 \mathrm{~h}$ compared with that in the vehicle group $(n=6$, $p<0.05$ ). (2) Preventive effects of pharmacological Wnt blockers. (f) On day 6,12 rats were divided into two groups that received an intraperitoneal injection of vehicle or XAV-939 (3 mg/kg) twice daily on days 6-14 (hatched box) during the pain development period by PAC $(n=6)$. The multiple injections of XAV-939 prevented the induction of PINP by day $40(n=6, p<0.05)$. (g) On day 6,12 rats were divided into two groups that received an intraperitoneal injection of vehicle or LGK-974 (2 mg/kg) twice daily on days 6-14 (hatched box) during the pain development period by PAC $(n=6)$. The multiple injections of LGK-974 prevented the induction of PINP by day $40(n=6, p<0.05)$. The data are expressed as the means and standard errors of the means. The asterisks indicate values that are significantly different $(n=6, p<0.05)$ from the corresponding values for the vehicle group, as determined by a two-way repeated-measures analysis of variance with one repeated factor (time), followed by the Tukey post hoc test

\section{Co-localization of $\beta$-Catenin in Human DRGs}

An immunohistochemical analysis revealed that the expression of active $\beta$-catenin in the painful area was expressed in neurons (Fig. 5(d)), especially in CGRP-expressing neurons (Fig. 5(1)) and GFAP-expressing satellite cells (Fig. 5(t)). The intensity of $\beta$-catenin in painful DRG was higher than that in unpainful DRG (103.2 \pm 27.9 of painful DRG vs $41.8 \pm 16.9$ of unpainful DRG, mean \pm standard deviation, $n=3$ sections, $p<0.05$, Fig. 5(a) vs 5(e)). These data indicate that $\beta$-catenin was co-localized in human DRG neuron cells, including CGRP-expressing cells and satellite cells.

\section{Discussion}

In this study, we showed that XAV-934 and LGK-974 produced preventive and analgesic effects by inhibiting the release of Wnt3a, Wnt10a, and active $\beta$-catenin in the DRG. In addition, PAC increased the expression of Axin2 in Axin2LacZ mice. Furthermore, active $\beta$-catenin was co-localized with DRG neurons, including CGRP-expressing neurons and satellite cells in human and rat DRG.

The results of previous studies of neuropathic pain and the Wnt pathway indicated that nerve injury-induced neuropathic pain in spinal nerve ligation and chronic constriction injury model was decreased by (1) overexpression of secreted Frizzled-related protein 1 in the spinal cord, (2) the suppression of the non-canonical signaling pathway, and (3) intrathecal injection of $\mathrm{Wnt} / \beta$-catenin pathway inhibitor and transcription factor 4 small interfering RNA [10-12, 25-28]. Recently, intrathecal injections of pharmacological Wnt inhibitors, including LGK974, NSC668036, and PNU76454, showed analgesic effects in PAC-induced neuropathy by inhibiting inflammation in rats [28]. PAC cannot penetrate the blood-brain barrier, accumulate in DRGs, and cause damage to the sciatic nerve, nerve endings, and DRGs during the development of pain behavior [29, 30]. Therefore, DRG may be a major site for the induction of CINP. Our study is the first to report the preventive effects of the systemic administration of canonical Wnt blockers and that Wnt3a, Wnt10a, and $\beta$ catenin may be major modulators for the induction of CINP in the DRG.

In our study, among 19 Wnt ligands, PAC increased the release of the Wnt ligands Wnt3a and Wnt10a. We recently found that PAC increases reactive oxygen species (ROS) in the rat DRG [15]. Furthermore, ROS-activated hypoxia-inducible factors directly transactivate $\mathrm{Wnt} 2 \mathrm{~b}$ for $\beta$-catenin stabilization in the regenerating intestine [31], indicating that PAC increases canonical Wnt ligands via the ROS-activated hypoxia-inducible factors axis in the DRG. In addition, $\beta$ catenin was co-localized with DRG neuron cells, CGRPexpressing neuron cells, and satellite cells in human and rat DRG. PAC increased the protein levels of phospho-NF-kB, MCP-1, and IL- $1 \beta$ in the DRG. Furthermore, phospho$\mathrm{NF}-\mathrm{kB}$, an activated form of NF- $\mathrm{kB}$, was translocated into the nucleus from the cytosol and produced various inflammatory cytokines and chemokines, such as TNF- $\alpha$, IL- $1 \beta$, and MCP-1, which can produce pain behaviors [32]. MCP-1 was expressed in the DRG, spinal cord, and astrocytes; was bound to chemokine CC motif receptor-1 in microglia, astrocytes, and the spinal cord; and was upregulated in the spinal cord in various pain conditions, including spinal nerve ligation, spinal cord contusion injury, and bone cancer pain [33-35]. We observed reductions in PAC-upregulated TNF- $\alpha$, IL-1 $\beta$, MCP-1, Wnt3a, and Wnt10a by XAV-939, which indicates that $\beta$-catenin contributes to the PAC-induced expression of these inflammatory cytokines. $\beta$-Catenin was found to transactivate TNF- $\alpha$, IL- $1 \beta$, and MCP-1 in the spinal cord, chondrocytes, and human bronchial epithelial cells [36-38]. Thus, it is highly likely that it mediates the PAC-induced 


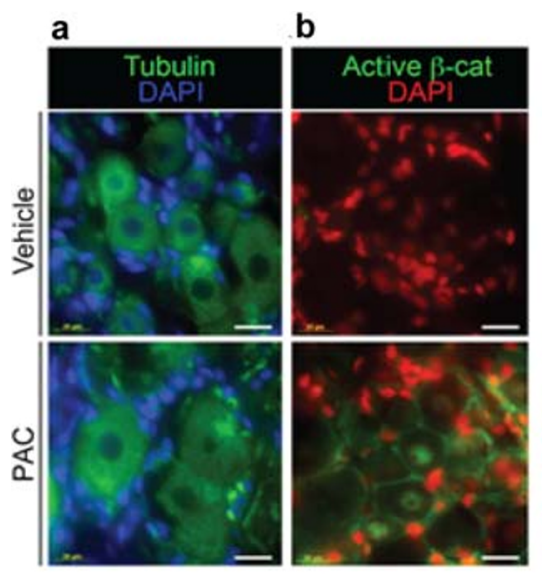

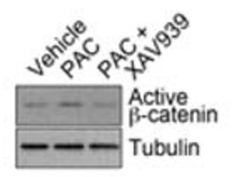

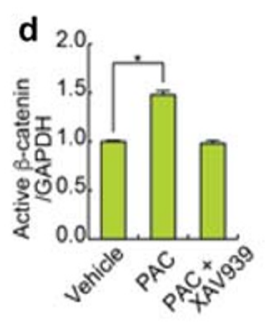

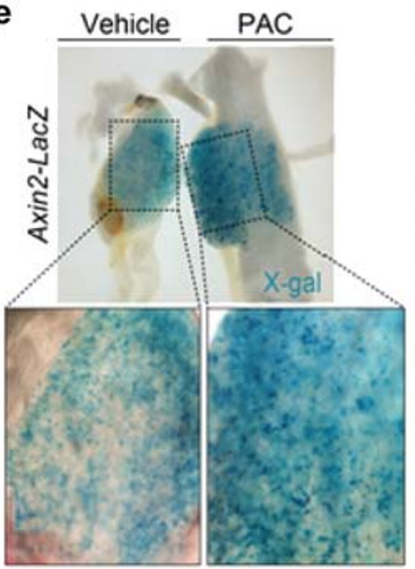

f Vehicle PAC

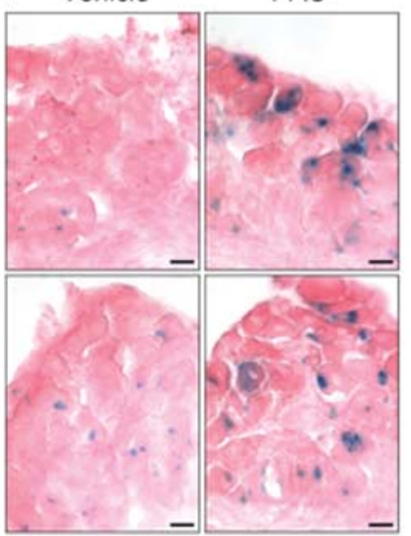

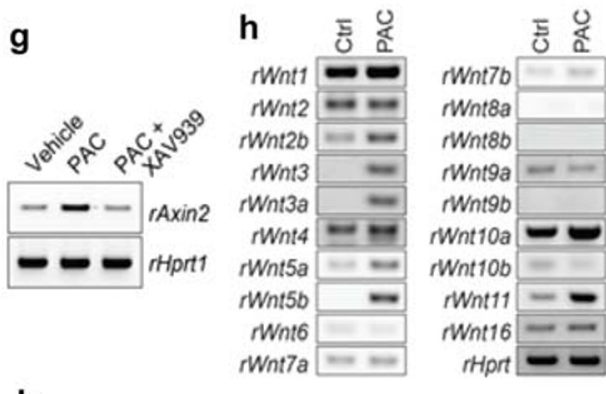

\section{i}
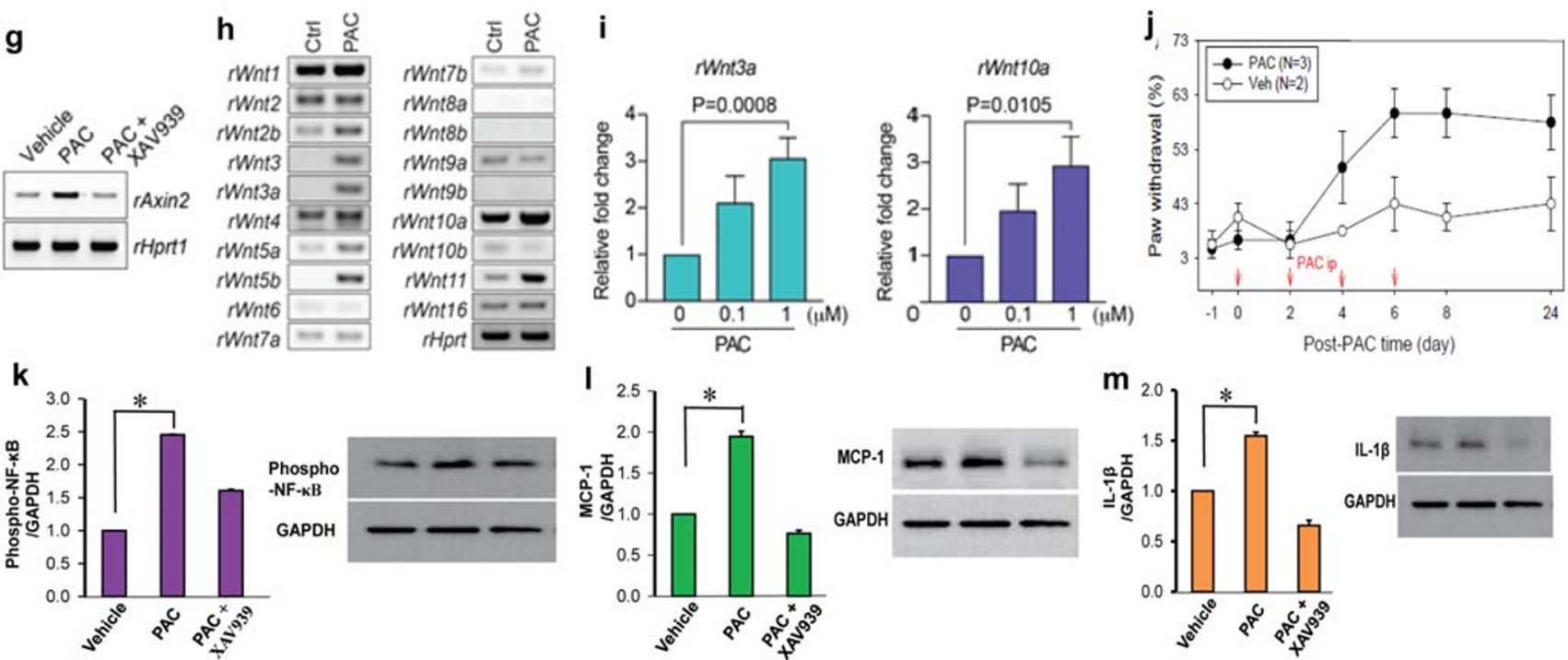

Fig. 3 Paclitaxel (PAC)-activated Wnt/ $\beta$-catenin signaling and inflammatory mediators. (a) No changes in tubulin dynamics after treatment with PAC. (b) Immunofluorescent staining of the L5 DRG (vehicle control vs. PAC). Thick tubule bundles were observed in PAC-injected DRG. DAPI: nuclear counterstaining. PAC-activated $\beta$-catenin in the DRG. Immunofluorescent staining of the L5 DRG for active $\beta$-catenin. Active $\beta$-catenin (green) was located in the nucleus of neurons and satellite cells in the rat DRG. (c) PAC-Western blot analysis of the L5 DRG for active $\beta$-catenin. (d) Quantification of active $\beta$-catenin in the DRG. Note that PAC increased the levels of active $\beta$-catenin in the DRG and XAV-939 subsequently decreased the protein levels $(n=3, p<0.05)$. (e, f) X-gal (5bromo-4-chloro-3-indolyl- $\beta$-D-galacto-pyranoside) staining of Axin2LacZ mice (vehicle vs. PAC, $n=3$ ). Whole-mount staining (e); cryosectioned staining of X-gal and nuclear FastRed (f). (g) Upregulation of rAxin2 by PAC. Semi-QT-RT-PCR of rat DRG for rAxin2 expression analysis. (h) Upregulation of canonical Wnt ligands

by PAC in rat L4-6 DRG, as determined by semi-QT-RT-PCR analysis. (i) Release of $r W n t 3 a$ and $r W n t 10 a$ in the primary DRG cell culture by PAC $(n=3)$. (j) PAC was injected intraperitoneally on days 0,2 , 4, and 6 in Axin2-LacZ mice, and the mechanical threshold was measured. Note that PAC increased the paw withdrawal response in mice $(n=2$ or 3$)$. ( $\mathrm{k}-$ $\mathrm{m})$ Western blot analysis showing the expression of phospho-NF-kB, MCP-1, and IL-1 $\beta$ in L1-6 DRGs after the injection of vehicle or PAC on day 17. XAV-939 was intraperitoneally injected twice daily on day 16 and injected in the morning on day 17. The DRGs of PAC+XAV939 were collected 1-2 $\mathrm{h}$ after the last injection on day 17. Quantification of phospho-NF-kB (65 kDa), MCP-1 (12 kDa), and IL-1 $\beta(17 / 31 \mathrm{kDa})$ in DRG. PAC increased the levels of phospho-NF-kB (k), MCP-1 (l), and $\mathrm{IL}-1 \beta(\mathrm{m})$ in the rat DRG $(n=3, p<0.05)$; XAV-939 decreased these protein levels. Data are the means with standard deviations for three rats, and asterisks indicate significant differences $(n=3, p<0.05)$ compared with the vehicle group, as determined by the Mann-Whitney $U$ test

upregulation of inflammatory cytokines. Our results suggest a model for the induction of CINP. Chemotherapy released Wnt3a and Wnt10a and then released active $\beta$-catenin, which induced CINP. Wnt blockers, including XAV-939 and LGK974, prevented CINP.

In our study, active $\beta$-catenin was expressed in CGRPexpressing neurons in the painful DRG in rats and humans. Most CGRP-expressing DRG neurons are small, but some are

medium and a few are large, indicating that while most CGRP afferent axons are unmyelinated, some are myelinated. Therefore, the major CGRP-expressing DRG neurons are $\mathrm{C}$ fiber and some are $A \beta$ and $A \delta$ fibers [39-42]. In addition, CGRP is reported to co-localize with various pain-related peptides, including substance $\mathrm{P}$, galanin, cholecystokinin, and vasoactive intestinal polypeptide in the DRG $[43,44]$. Neuropathic pain is reported to be involved in $C, A \beta$, and 

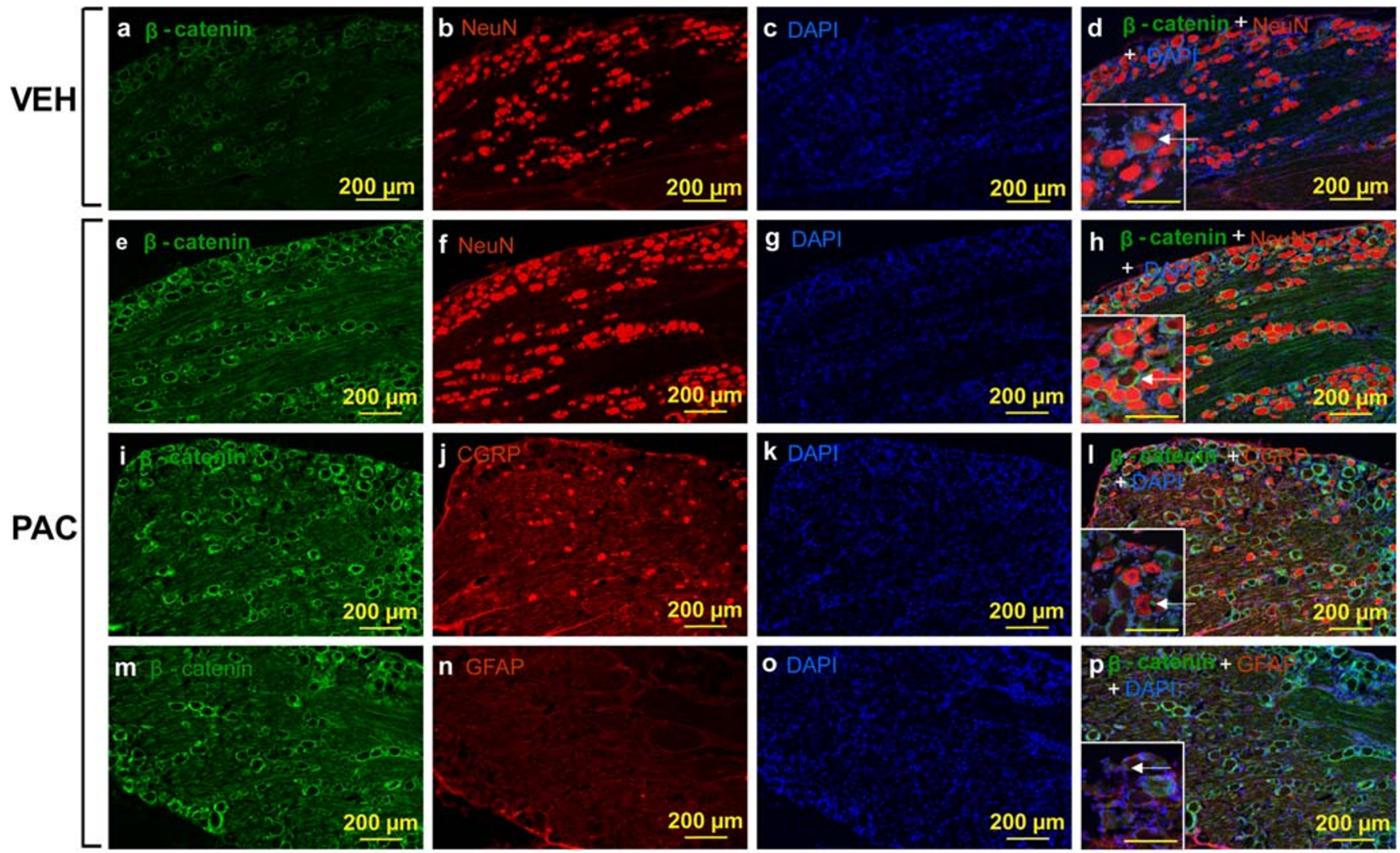

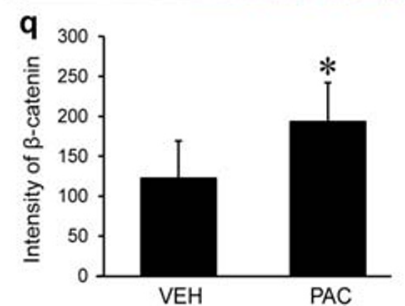

Fig. 4 Co-localization of $\beta$-catenin in neurons, including CGRPexpressing neurons and satellite cells, in rat DRG. Paclitaxel (PAC, $2 \mathrm{mg} / \mathrm{kg}$ ) or vehicle (VEH, 4\% dimethyl sulfoxide and 4\% Tween 80 in saline, $1 \mathrm{ml} / \mathrm{kg}$ ) was injected intraperitoneally on days $0,2,4$, and 6 in the PAC group $(n=3)$ and VEH group $(n=3)$. On day 16 after the first PAC or vehicle injection, the L5 DRG was obtained, post-fixed, cryoprotected, cryosectioned, and mounted on slides. (a) DRG was isolated from the VEH group. Note that the DRG neurons from the VEH group expressed $\beta$-catenin (green) as a canonical Wnt signaling marker. (b) NeuN (red) in the DRG from the VEH group. (c) DAPI (blue) in the DRG from the VEH group. (d) $\beta$-Catenin (green), NeuN (red), and DAPI (blue) in the DRG from the VEH group. Note that $\beta$-catenin was expressed in neurons in the DRG. Arrow indicates $\beta$-catenin with NeuN in DRG. Scale bar in the inset was $100 \mu \mathrm{m}$. (e) $\beta$-Catenin (green) in the DRG from the PAC group. (f) NeuN (red) in the DRG from the PAC group. (g) DAPI (blue) in the DRG from the PAC group. (h) $\beta$-Catenin (green), NeuN (red), and DAPI (blue) in the DRG from the PAC group. Note that $\beta$-catenin was expressed in neurons in the DRG. Arrow indicates $\beta$-catenin with

$\mathrm{A} \delta$ fibers [45]. Therefore, $\beta$-catenin is likely to associate with CINP through its presence in $\mathrm{C}, \mathrm{A} \beta$, and $\mathrm{A} \delta$ fibers and its interaction with other peptides.

There are currently no Food and Drug Administrationapproved regimens for CINP prevention; thus, our results may shed light on the development of promising therapeutic
NeuN in DRG. Scale bar in the inset was $100 \mu \mathrm{m}$. (i) $\beta$-Catenin (green) in the DRG from the PAC group. (j) CGRP (red) in the DRG from the PAC group. (k) DAPI (blue) in the DRG from the PAC group. (l) $\beta$ Catenin (green), CGRP (red), and DAPI (blue) in the DRG from the PAC group. $\beta$-Catenin was expressed in CGRP-expressing neurons in the DRG. Arrow indicates $\beta$-catenin with CGRP in the DRG. Scale bar in the inset is $100 \mu \mathrm{m}$. (m) $\beta$-Catenin (green) in the DRG from the PAC group. (n) GFAP (red) in the DRG from the PAC group. (o) DAPI (blue) in the DRG from the PAC group. (p) $\beta$-Catenin (green), GFAP (red), and DAPI (blue) in the DRG from the PAC group. $\beta$-Catenin was expressed in satellite cells in the DRG. Arrow indicates $\beta$-catenin with GFAP in the DRG. Scale bar in the inset was $100 \mu \mathrm{m}$. (q) The intensity of $\beta$-catenin in the DRG from the VEH and PAC groups. PAC increased the intensity of $\beta$-catenin in the rat DRG $(n=3, p<0.05)$. Data are the means with standard deviations for three rats. Asterisks indicate significant differences $(n=3, p<0.05)$ compared with the vehicle group, as determined by the Mann-Whitney $U$ test. Scale bars, $200 \mu \mathrm{m}$

applications for CINP. In addition, many studies reported the anti-tumorigenic effects of XAV-939, LGK-974, and iCRT14 on Wnt signaling-associated human cancers [46-49]. Several clinical studies have focused on the therapeutic impact of LGK-974 in melanoma and squamous cell, triple-negative breast, head and neck, pancreatic, cervical, esophageal, and 
[1] Co-localization of $\beta$-catenin with NeuN in human DRG
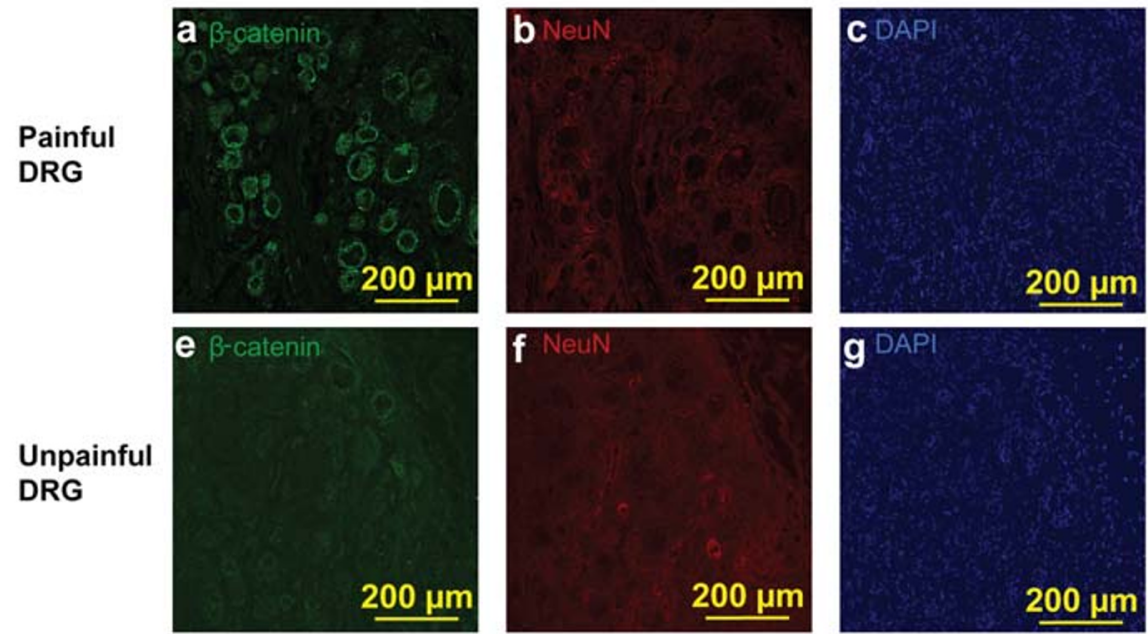

[2] Co-localization of $\beta$-catenin with CGRP in human DRG
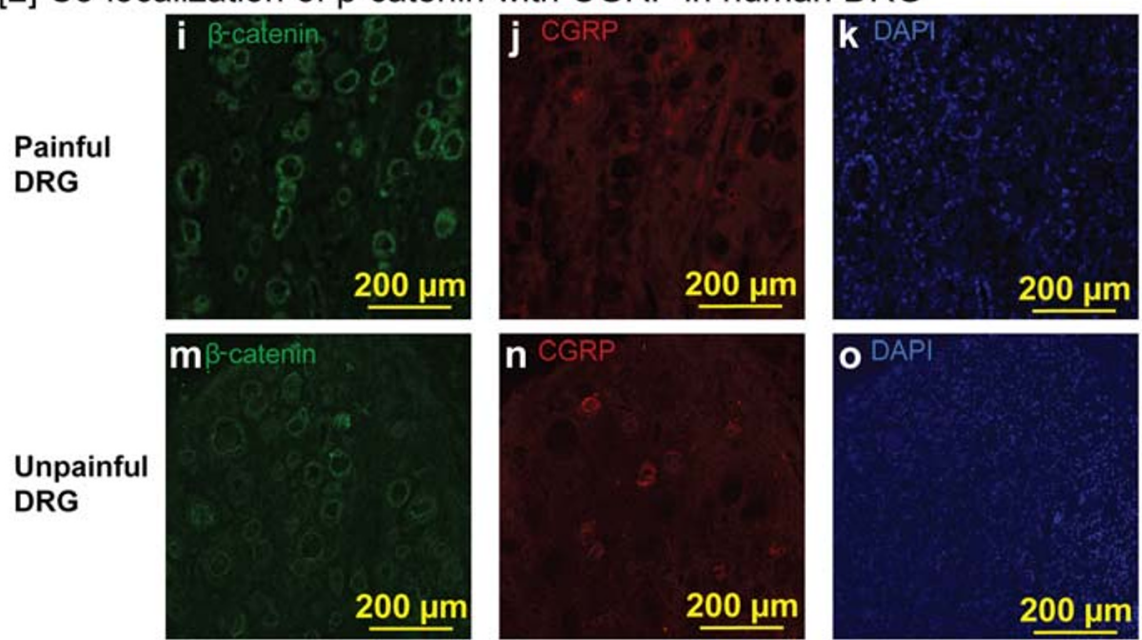

[3] Co-localization of $\beta$-catenin with GFAP in human DRG
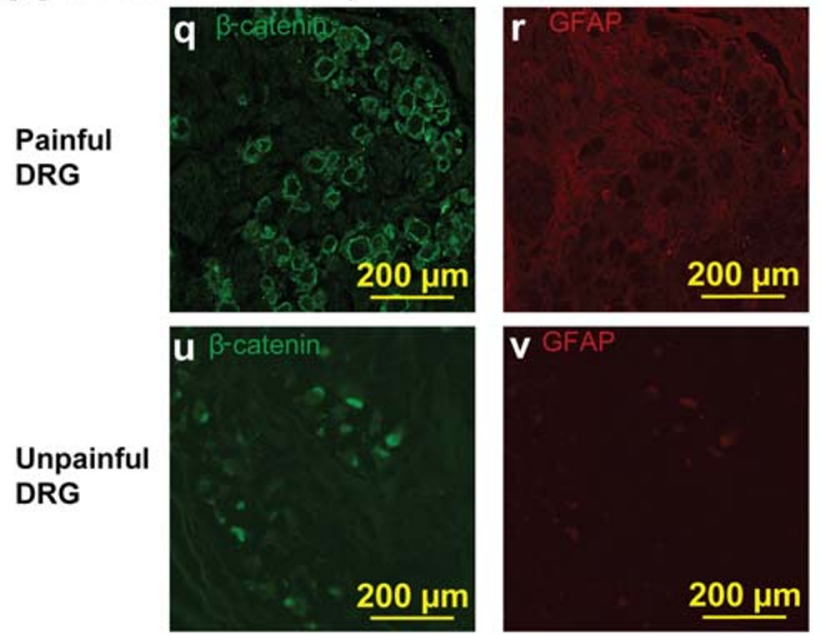
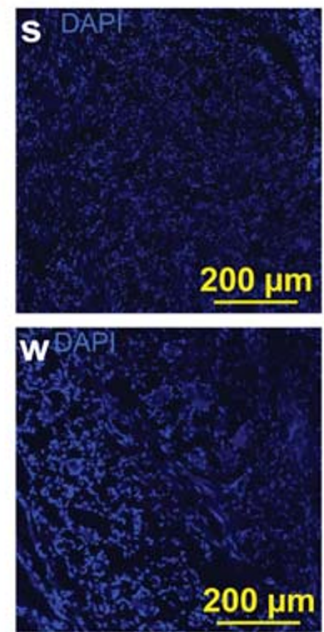
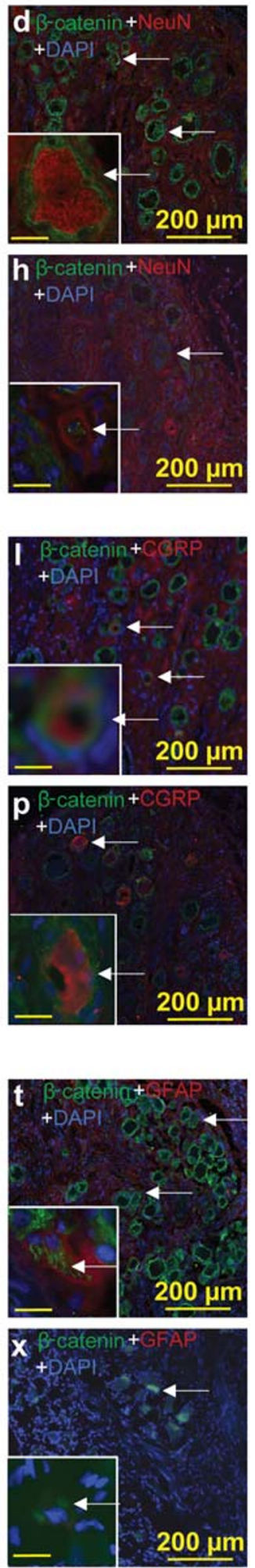

lung cancers (www.clinicaltrials.gov). Therefore, Wnt signaling blockers, in combination with other neuropathic pain-inducing chemotherapeutic drugs, may decrease the intensity, duration, and prevalence of pain and anticancer effects, which is the best choice for cancer patients and survivors. Thus, targeting of $\mathrm{Wnt} / \beta$-catenin signaling may be an effective regimen for both CINP and Wnt signalingassociated cancer; this should be addressed in future studies. 
Fig. 5 Co-localization of $\beta$-catenin in DRG neurons and satellite cells in human DRG. (1) Co-localization of $\beta$-catenin with NeuN in human DRG. (a) The DRG was isolated from the pain area of a patient. Note that the DRG neurons from this area had increased expression of $\beta$ catenin (green) as a canonical Wnt signaling marker. (b) NeuN (red) in the DRG from the pain area. (c) DAPI (blue) in the DRG from the pain area. (d) $\beta$-Catenin (green), NeuN (red), and DAPI (blue) in the DRG from the pain area. $\beta$-Catenin was expressed in neurons. Arrows indicate $\beta$-catenin with NeuN. Scale bar in the inset was $20 \mu \mathrm{m}$. (e) DRG was isolated from outside the pain area of a patient. Note that the DRG neurons from outside the pain area expressed low levels $\beta$-catenin (green) compared with that in the pain area (a). (f) NeuN (red) in the DRG from outside the pain area. (g) DAPI (blue) in the DRG from outside the pain area. (h) $\beta$-Catenin (green), NeuN (red), and DAPI (blue) in the DRG from outside the pain area. Scale bar in the inset was $20 \mu \mathrm{m}$. (2) Colocalization of $\beta$-catenin with CGRP in human DRG. (i) The DRG was isolated from the pain area of a patient. Note that the DRG neurons from the pain area had increased expression of $\beta$-catenin (green) compared with that in the unpainful area (m). (j) CGRP (red) in the DRG from the pain area. (k) DAPI (blue) in the DRG from the pain area. (l) $\beta$-Catenin (green), CGRP (red), and DAPI (blue) in the DRG from the pain area. Arrows indicate $\beta$-catenin with CGRP. $\beta$-Catenin was expressed in CGRP-expressing neurons. Scale bar in the inset was $20 \mu \mathrm{m}$. (m) The DRG was isolated from outside the pain area of a patient. $\beta$-Catenin (green) in the DRG from outside the pain area. (n) CGRP (red) in the DRG from outside the pain area. (o) DAPI (blue) in the DRG from outside the pain area. (p) $\beta$-Catenin (green), CGRP (red), and DAPI (blue) in the DRG from outside the pain area. Arrows indicate $\beta$ catenin with CGRP. Scale bar in the inset was $20 \mu \mathrm{m}$. (3) Colocalization of $\beta$-catenin with GFAP in human DRG. (q) DRG was isolated from the pain area of a patient. Note that the DRG neurons from the pain area had increased expression of $\beta$-catenin (green) compared with that in the unpainful area (u). (r) The GFAP (red) in the DRG from the pain area. (s) DAPI (blue) in the DRG from the pain area. (t) $\beta$-Catenin (green), GFAP (red), and DAPI (blue) in the DRG from the pain area. Arrows indicate $\beta$-catenin with GFAP. $\beta$-Catenin was expressed in GFAP-expressing satellite cells. Scale bar in the inset was $20 \mu \mathrm{m}$. (u) The DRG was isolated from outside the pain area of a patient. $\beta$-Catenin (green) in the DRG from outside the pain area. (v) GFAP (red) in the DRG from outside the pain area. (w) DAPI (blue) in the DRG from outside the pain area. (x) $\beta$-Catenin (green), GFAP (red), and DAPI (blue) in the DRG from outside the pain area. Arrows indicate $\beta$ catenin with GFAP. Scale bar in the inset is $20 \mu \mathrm{m}$. Scale bars, $200 \mu \mathrm{m}$

This study has clear limitations. First, we did not measure pain in tumor-bearing animals. CINP animal models have been developed using normal animals by the injection of chemotherapeutic drugs. Therefore, we developed a CINP model of tumor-bearing animals. Second, we used PAC as chemotherapy. The mechanisms of CINP vary greatly on the basis of several factors, especially chemotherapy type.

Recently, sex dimorphism has been reported in various CINP models, including rats and mice. Stockstill et al. reported that sphingosine-1-phosphate receptor subtype 1 antagonists and $\mathrm{A} 3$ adenosine receptor subtype agonists mitigate paclitaxel- and oxaliplatin-induced neuropathic pain in male and female rodents but failed to block or reverse bortezomibinduced neuropathic pain in females [50]. Ferrari et al. also reported that sexual dimorphism contributes to the dependence of paclitaxel-induced hyperalgesia on the two main neuroendocrine stress axis mediators, corticosterone and epinephrine, acting on nociceptors at their cognate receptors ( $\beta_{2}$-adrenergic and glucocorticoid, respectively) [51]. In addition, Luo et al. reported that macrophage Toll-like receptor 9 signaling promotes paclitaxel-induced neuropathic pain in only male mice, not female mice, and resolvin D5 inhibits paclitaxel-induced neuropathic pain and inflammatory pain in only male mice, not female mice $[52,53]$. We will identify analgesia of Wnt blockers in CINP in female mice in our next project.

We conclude that Wnt3a, Wnt10a, and $\beta$-catenin play a crucial role in the induction and maintenance of CINP, and $\mathrm{Wnt} / \beta$-catenin signaling blockers show preventive and analgesic effects in CINP rats. In addition, $\beta$-catenin was colocalized with DRG neurons, CGRP-expressing DRG neuron, and satellite cells in human and rat DRG. Therefore, $\mathrm{Wnt} / \beta$ catenin signaling may be an important target for both CINP and cancer.

Acknowledgments This work was supported by the Cancer Prevention Research Institute of Texas (RP140563 to J-IP) and by grants from the Peggy and Avinash Ahuja Foundation and the Helen Buchanan and Stanley Joseph Seeger Endowment at The University of Texas MD Anderson Cancer Center (to SA) and CA200263, HEB Professor in Cancer Research, The Thomson Family Foundation Initiative, and NS111929 (to PMD). This work was also supported by the Collaborative Genome Program for Fostering New Post-Genome Industry (to S-WL; NRF-2017M3C9A5031597) of the National Research Foundation, funded by the Korean Ministry of Science and ICT, and partially supported by the Brain Research Program through the National Research Foundation, funded by the Ministry of Science and ICT (to M-SK; NRF-2017M3C7A1027472). The authors thank Ann Sutton (Editing Services, Research Medical Library, The University of Texas MD Anderson Cancer Center) for the editorial assistance.

Required Author Forms Disclosure forms provided by the authors are available with the online version of this article.

\section{Compliance with Ethical Standards}

Conflict of Interest The authors declare that they have no competing interests.

\section{References}

1. Massey RL, Kim HK, Abdi S. Brief review: chemotherapy-induced painful peripheral neuropathy (CIPPN): current status and future directions. Can J Anaesth 2014;61(8):754-62.

2. Nusse R, Clevers H. Wnt/beta-Catenin Signaling, Disease, and Emerging Therapeutic Modalities. Cell. 2017;169(6):985-99.

3. Oliva CA, Montecinos-Oliva C, Inestrosa NC. Wnt Signaling in the Central Nervous System: New Insights in Health and Disease. Prog Mol Biol Transl Sci 2018;153:81-130.

4. Rosso SB, Sussman D, Wynshaw-Boris A, et al. Wnt signaling through Dishevelled, Rac and JNK regulates dendritic development. Nat Neurosci 2005;8(1):34-42.

5. Rosso SB, Inestrosa NC. WNT signaling in neuronal maturation and synaptogenesis. Front Cell Neurosci 2013;7:103. 
6. Munoz FJ, Godoy JA, Cerpa W, et al. Wnt-5a increases NO and modulates NMDA receptor in rat hippocampal neurons. Biochem Biophys Res Commun 2014;444(2):189-94.

7. Clevers H, Nusse R. Wnt/beta-catenin signaling and disease. Cell. 2012;149(6):1192-205.

8. Willert K, Nusse R. Wnt proteins. Cold Spring Harb Perspect Biol 2012;4(9):a007864.

9. Anastas JN, Moon RT. WNT signalling pathways as therapeutic targets in cancer. Nat Rev Cancer 2013;13(1):11-26.

10. Tang J, Ji Q, Jin L, et al. Secreted frizzled-related protein 1 regulates the progression of neuropathic pain in mice following spinal nerve ligation. J Cell Physiol 2018;233(8):5815-22.

11. Yuan S, Shi Y, Guo K, et al. Nucleoside Reverse Transcriptase Inhibitors (NRTIs) Induce Pathological Pain through Wnt5aMediated Neuroinflammation in Aging Mice. J NeuroImmune Pharmacol 2018;13(2):230-36.

12. $\mathrm{Xu} \mathrm{Z}$, Chen $\mathrm{Y}, \mathrm{Yu}$ J, et al. TCF4 Mediates the Maintenance of Neuropathic Pain Through Wnt/beta-Catenin Signaling Following Peripheral Nerve Injury in Rats. J Mol Neurosci 2015;56(2):397-408.

13. Kim HK, Zhang YP, Gwak YS, et al. Phenyl N-tert-butylnitrone, a free radical scavenger, reduces mechanical allodynia in chemotherapy-induced neuropathic pain in rats. Anesthesiology. 2010;112(2):432-9.

14. Kim HK, Hwang SH, Oh E, et al. Rolipram, a Selective Phosphodiesterase 4 Inhibitor, Ameliorates Mechanical Hyperalgesia in a Rat Model of Chemotherapy-Induced Neuropathic Pain through Inhibition of Inflammatory Cytokines in the Dorsal Root Ganglion. Front Pharmacol. 2017;8:885.

15. Kim HK, Hwang SH, Abdi S. Tempol Ameliorates and Prevents Mechanical Hyperalgesia in a Rat Model of ChemotherapyInduced Neuropathic Pain. Front Pharmacol 2016;7:532.

16. Uchida H, Nagai J, Ueda H. Lysophosphatidic acid and its receptors LPA1 and LPA3 mediate paclitaxel-induced neuropathic pain in mice. Mol Pain 2014;10:71.

17. Dixon WJ. Efficient analysis of experimental observations. Annu Rev Pharmacol Toxicol 1980;20:441-62.

18. Bae C, Wang J, Shim HS, et al. Mitochondrial superoxide increases excitatory synaptic strength in spinal dorsal horn neurons of neuropathic mice. Mol Pain 2018;14:1744806918797032.

19. Aebersold R, Mann M. Mass spectrometry-based proteomics. Nature. 2003;422(6928):198-207.

20. Aebersold R, Mann M. Mass-spectrometric exploration of proteome structure and function. Nature. 2016;537(7620):347-55.

21. Lee H, Mun DG, So JE, et al. Efficient Exploitation of Separation Space in Two-Dimensional Liquid Chromatography System for Comprehensive and Efficient Proteomic Analyses. Anal Chem 2016;88(23):11734-41.

22. Kim E, Hwang SH, Kim HK, et al. Losartan, an Angiotensin II Type 1 Receptor Antagonist, Alleviates Mechanical Hyperalgesia in a Rat Model of Chemotherapy-Induced Neuropathic Pain by Inhibiting Inflammatory Cytokines in the Dorsal Root Ganglia. Mol Neurobiol 2019;56(11):7408-19.

23. Li Y, North RY, Rhines LD, et al. DRG Voltage-Gated Sodium Channel 1.7 Is Upregulated in Paclitaxel-Induced Neuropathy in Rats and in Humans with Neuropathic Pain. J Neurosci 2018;38(5):1124-36

24. Lustig B, Jerchow B, Sachs M, et al. Negative feedback loop of Wnt signaling through upregulation of conductin/axin2 in colorectal and liver tumors. Mol Cell Biol 2002;22(4):1184-93.

25. Liu S, Liu YP, Huang ZJ, et al. Wnt/Ryk signaling contributes to neuropathic pain by regulating sensory neuron excitability and spinal synaptic plasticity in rats. Pain. 2015;156(12):2572-84.

26. Yang QO, Yang WJ, Li J, et al. Ryk receptors on unmyelinated nerve fibers mediate excitatory synaptic transmission and CCL2 release during neuropathic pain induced by peripheral nerve injury. Mol Pain 2017;13:1744806917709372.

27. Itokazu T, Hayano Y, Takahashi R, et al. Involvement of Wnt/betacatenin signaling in the development of neuropathic pain. Neurosci Res 2014;79:34-40.

28. Resham K, Sharma SS. Pharmacological interventions targeting Wnt/beta-catenin signaling pathway attenuate paclitaxel-induced peripheral neuropathy. Eur J Pharmacol 2019;864:172714.

29. Cavaletti G, Cavalletti E, Oggioni N, et al. Distribution of paclitaxel within the nervous system of the rat after repeated intravenous administration. Neurotoxicology. 2000;21(3):389-93.

30. Peters CM, Jimenez-Andrade JM, Kuskowski MA, et al. An evolving cellular pathology occurs in dorsal root ganglia, peripheral nerve and spinal cord following intravenous administration of paclitaxel in the rat. Brain Res 2007;1168:46-59.

31. Suh HN, Kim MJ, Jung YS, et al. Quiescence Exit of Tert(+) Stem Cells by Wnt/beta-Catenin Is Indispensable for Intestinal Regeneration. Cell Rep 2017;21(9):2571-84.

32. Deshpande R, Khalili H, Pergolizzi RG, et al. Estradiol downregulates LPS-induced cytokine production and NFkB activation in murine macrophages. Am J Reprod Immunol 1997;38(1):46-54.

33. Jeon SM, Lee KM, Cho HJ. Expression of monocyte chemoattractant protein-1 in rat dorsal root ganglia and spinal cord in experimental models of neuropathic pain. Brain Res 2009;1251: $103-11$.

34. Wang $\mathrm{Y}$, Ni H, Li H, et al. Nuclear factor kappa B regulated monocyte chemoattractant protein-1/chemokine CC motif receptor- 2 expressing in spinal cord contributes to the maintenance of cancerinduced bone pain in rats. Mol Pain 2018;14:1744806918788681.

35. Kiguchi N, Ding H, Peters CM, et al. Altered expression of glial markers, chemokines, and opioid receptors in the spinal cord of type 2 diabetic monkeys. Biochim Biophys Acta Mol basis Dis 2017;1863(1):274-83.

36. Zhang YK, Huang ZJ, Liu S, et al. WNT signaling underlies the pathogenesis of neuropathic pain in rodents. J Clin Invest 2013;123(5):2268-86.

37. Chang Y, Wang X, Sun Z, et al. Inflammatory cytokine of IL-1 beta is involved in T-2 toxin-triggered chondrocyte injury and metabolism imbalance by the activation of Wnt/beta-catenin signaling. Mol Immunol 2017;91:195-201.

38. Jang J, Jung Y, Kim Y, et al. LPS-induced inflammatory response is suppressed by Wnt inhibitors, Dickkopf-1 and LGK974. Sci Rep 2017;7:41612.

39. Carlton SM, McNeill DL, Chung K, et al. A light and electron microscopic level analysis of calcitonin gene-related peptide (CGRP) in the spinal cord of the primate: an immunohistochemical study. Neurosci Lett 1987;82(2):145-50.

40. Harmann PA, Chung K, Briner RP, et al. Calcitonin gene-related peptide (CGRP) in the human spinal cord: a light and electron microscopic analysis. J Comp Neurol 1988;269(3):371-80.

41. Ishida-Yamamoto A, Senba E, Tohyama M. Calcitonin generelated peptide- and substance P-immunoreactive nerve fibers in Meissner's corpuscles of rats: an immunohistochemical analysis. Brain Res 1988;453(1-2):362-6.

42. Lawson SN, Perry MJ, Prabhakar E, et al. Primary sensory neurones: neurofilament, neuropeptides, and conduction velocity. Brain Res Bull 1993;30(3-4):239-43.

43. Arciszewski MB. Distribution of calcitonin gene-related peptide (CGRP), substance P (SP) and galanin (GAL) immunoreactive nerve fibers in the seminal vesicle and prostate of the male sheep. Ann Anat 2004;186(1):83-7.

44. Riesco N, Cernuda-Morollon E, Pascual J. Neuropeptides as a Marker for Chronic Headache. Curr Pain Headache Rep 2017;21(4):18. 
45. Shim HS, Bae C, Wang J, et al. Peripheral and central oxidative stress in chemotherapy-induced neuropathic pain. Mol Pain 2019;15:1744806919840098.

46. Liu J, Pan S, Hsieh MH, et al. Targeting Wnt-driven cancer through the inhibition of Porcupine by LGK974. Proc Natl Acad Sci U S A 2013;110(50):20224-9.

47. Wickstrom M, Dyberg C, Milosevic J, et al. Wnt/beta-catenin pathway regulates MGMT gene expression in cancer and inhibition of Wnt signalling prevents chemoresistance. Nat Commun 2015;6: 8904.

48. Zimmerli D, Cecconi V, Valenta T, et al. WNT ligands control initiation and progression of human papillomavirus-driven squamous cell carcinoma. Oncogene. 2018;37(27):3753-62.

49. Huang SM, Mishina YM, Liu S, et al. Tankyrase inhibition stabilizes axin and antagonizes Wnt signalling. Nature. 2009;461(7264): 614-20.

50. Stockstill K, Wahlman C, Braden K, et al. Sexually dimorphic therapeutic response in bortezomib-induced neuropathic pain reveals altered pain physiology in female rodents. Pain. 2020;161(1):177-84.

51. Ferrari LF, Araldi D, Green PG, et al. Marked sexual dimorphism in neuroendocrine mechanisms for the exacerbation of paclitaxelinduced painful peripheral neuropathy by stress. Pain. 2020;161(4):865-74.

52. Luo X, Huh Y, Bang S, et al. Macrophage Toll-like Receptor 9 Contributes to Chemotherapy-Induced Neuropathic Pain in Male Mice. J Neurosci 2019;39(35):6848-64.

53. Luo X, Gu Y, Tao X, et al. Resolvin D5 Inhibits Neuropathic and Inflammatory Pain in Male But Not Female Mice: Distinct Actions of D-Series Resolvins in Chemotherapy-Induced Peripheral Neuropathy. Front Pharmacol 2019;10:745.

Publisher's Note Springer Nature remains neutral with regard to jurisdictional claims in published maps and institutional affiliations. 